\title{
Analysis on Developing Practice and Model of Physical Training and Education
}

\author{
Qiangsong Deng \\ Jingchu University of Technology, Jingmen Hubei, 448000, China
}

Keywords: Physical education, Training teaching, Model.

\begin{abstract}
Deficiencies of sports training and teaching are unitary in content and forms, blindness in training process, etc, because lost enthusiasm and failure in chances of physical training. Therefore an optimal developmental teaching model is eagerly needed for the characteristics of sports physical education and actuarial requirement of students.. This article discussed the technique in the physical education, including discriminated training process; well organized training and favorable environment with music featured, as well as formulated the commonly used teaching models in physical education and the key features for performing physical training.
\end{abstract}

\section{Introduction}

Physical training can strength sports spirit, function in health and body building, strengthen willpower and interpersonal skills. The important factors for physical tainting in collage are application of scientific training equines according to the college students training hobbies, routine, tolerated training volume and physical conditions; a clear training goal should be constituted, to improve the efficiency to physical education ${ }^{[1]}$ 。 In another hand, the physical education have to master the relative teaching techniques, and rational use of teaching model, prevent from issues like unitary targets, absolute in both content and formulary and to assure the achievement of teacher-student interaction and improve the creativity, activity and motivation during the physical education. This article conducted an analysis on the developing parties and model for physical trading and education, for the purpose of improving students' physical health and intelligent level.

\section{The technique in physical training}

\section{Discriminated training process, well organized training.}

Discriminated training process means the entire training have to develop according to the athletic level and sports project, to ensure the redouble sport capacity and avoided excessive exercises and physical injuries. discriminated training process means the arrangement of the physical training match with student' s physical status and the character of individual sports, to the ensure the suitable loading capacity and prevented from injuries; the training time have to be arrange to the training characters and rule of the specific sports, appropriately increase completive train programs to enhance their interest of joint to own desired program. There many physical practice programs are availed in the colleague, if many student join the same physical practice at the same time may cause chaos for the PE teacher, so group trading can be adopted with full attention to different stages at the same sports group; for example, the key point for the beginning phases may be body training, while priority will switch into techniques and skills. In order to carry reasonable education, compurgation, games, and other teaching forms are good options according to the specific sports. ${ }^{[2]}$ 。 The computation can enchase their activities in initiative, enthusiasm, encourage them to challenge themselves and cone difficulties. For example, competition form can be used in following sports like gymnastics, aerobic, high and long jump, etc. Game form can edutainment, and avoid tiredness for the boring physical practice or training, including squat jumbo, tug and crouching fruit, etc. Decomposition teaching form suitable for difficult actions and key actions in competed sports like chuangquan, taijin and gymnastics, etc. 


\section{Introducing music element to create a good training environment.}

Music can made positive influence in both mental and emotional, introducing music into physical practice can help student switch into physical practice soon as possible. In the warming up period, the excursive intensity is low students are eon relaxed state in both physical and mental, some enjoyable and relaxing music work can be used. while music with good sense of rhythm and distinctive can help producing strong fighting moral and stimulate sport potential. For the ending of physical practice, some replacing smoothing music with long melodies will help stud dent relaxing after the intensive trainings. Moreover a good training environment will promote initiative of students and stimulate their desire and motivate To ensure effectiveness of the trading $^{[3]}$ in order to improve the effectiveness of the sport, teacher should equipped with relative sports equipment and facilities in a timely manner or carry simple alternative to the trading are or equipped with appropriate reform to ensure the effective of the training and the cooperation from students. Meanwhile observe the training process patiently, offering proper guidance' $s$ and communications to students, and then to build good interactive progress and ensure the achievement of the target. As well as flexible switching between training method, mining persona; potential and promote individual development

\section{Application of teaching model for physical training}

\section{The illusion training mode.}

Physical training is more than muscle excursive but also involved in motion perception. Motion perception can reflect back to brain about movement from both internal and external object, sense information about external objects like speed, shape side, distant and direction, etc, under the interference of color, shape and lighting condition may cause deviance to some of the motion perception that is illusion. Illusion can influence the physical practice in both positive and negative ways. Physical education should malaise the effect of positive illusion to promote improvement of student, even to gain better physical practice result. to maximize the effect of during the application the illusion training moved, teacher need to have good knowledge' $\mathrm{s}$ about the illusions in the physical practice and carry deep analysis for the teaching material and methods, replace undesirable interferences with some factor may cause positive illusions in the training period by the illusion laws. $^{[4]}$ first of all, the environmental factors can be adequately changed or enhanced, for example, during high jump training, the train area can be changed indoor to outdoor after the records tended to be stable, or the arrange a outdoor area with wide visual field with shorter- the- height illusion; with the help of this illusion, students can set for better record, may achieve it unconsciously. Another good example is enchanted the background on both side of the runways may result smaller the-space illations, which can make an thrill of running on wings by the runway space are narrowing down runways and flashing scene to backward, and scene backwards in a flesh. The another widely method of illusions are coloring illusion, which means the different shades of clout will interfere to the judgment of size weight, height, etc, for instant the light color will weigh the subject than darker color, even if the two injects are exactly the same in weight. this type of illusion are widely used in gymnastic training to relive mental pressure, pate the goats, boxes into bro not pale yellow, while the missiles like discus and shot ball painted into white. Place some heavy masses beside goats, boxes or drawing horizontal lines on the surface of boxes can "lower" the actual height and carry on the training in a relatively relaxed environment

\section{Microteaching training model.}

Microteaching training model is a physical practice though watching and analysis relative videos to discover and settle problem. Microteaching training model is unique in classification complex trading or sports and able to amplifier the training skills separately, is a very useful method in improving the physical practice. 。 It can help to unify the relationship between the individual and wholeness, and avoid fears for difficult sport. The application of this model can clearly the teaching objective, shorten demonstration time, keep records of the training process and results to ensure the accuracy and comprehensiveness of training and teaching evaluation, improve the effectiveness of physical practice. For instance, student can watch the videos of jumping process in high jump training, 
since the flash video can be stopped, repeated whenever necessary to build a clear imagery of high jump process in slow motion. ${ }^{[5]} \mathrm{A}$ the application of virtualization can acquaint student the relative techniques and motions, to avoid any injury caused by wrong movements. Teacher need to prepare a good video courseware in advance to enrich the train content and touring level, and conduct analysis on the results and feedback. Another example is in the trading, a microteaching goal have to be set , with detailed facial expression and body movement to ensure the achieving of the target. Then research information on line, stimulate students 'senses by perceptual audio materials, assemble motion imagery among students to ensure deep understand for key movements and train processes, while ensuring the attendee of every student in the training. In addition, tennis also widely use this teaching model; first of all recoding the trading process, organizing student to watch it and speaking freely, to make good understand of own disadvantages and communicate with each other. Teacher also can demonstrate commonly used steps such as left to right quick switch, acute, crash stop and change directions quickly, to teach them the migration of motor skills and accurately hit the ball from microteaching.

\section{Induction training model.}

Induction training model means students independently learn and prates the key action under influence of objective and subjective inducement. In this teaching model teacher need to consider the difficulty points, the character of the students to maximum the influence of the inducements. The right inducement should follow principles of easy to operate, vivid and intuitive and matched with training theme. So the student can be easily attracted by the inducer, and be more focused with it during the training. Moreover during the microteaching training mode, teacher should analysis the technique of the sports carefully, design suitable training programs and accurately controls training and duration timing to make the indumenta standardized and programmed. At present,the main applications of induction training model in physical education of colleagues are movement induction training and changed equipment induction training. Movement induction training is a transition training method which means student will learn the comprehensive action from a simple movement, like muscle stimulation proprioceptor can make then sense the changing of their body positions and postures during the movement and then make further understanding of key action. For example, the badminton training, the fist inducer is handling, the essential of performance of badminton can only be master by good handling. ${ }^{[6]}$ training will make student focused in the same direction at the same time with the ball and the exchanges of arm movement and gravity to coordinate the stroke. Changed equipment induction training means making appropriate changes to the sport equipment in natural and structures to lower the difficulty of the training. For example simplizing the equipement in high jump training by directly landed to the spong pad; take advantage of the spring board to increase the flight time and change positions in long jump training; use rubber bands instead of coclumns in the boar training to reduce fear ${ }^{[7]}$ And when training beginners and javelin whipping technique, softball wipping have to be induced by trainee,the javelin can be used after student has mastered the techniques to ensure the standardization of introduction training

\section{Conclusion}

In summary, the physical training education have to emphasis the improvement of student's knowledge and skill of sport, and optimize with the requirement of social demand, physical and mental characteristics of students, conduct suitable physical practice for specific sports, like physical psychological training, sport medicine train and Taijin quan ,etc. to assure the achievement of limb movement function of physical practice, college physical teacher need to emphasis the personal and professional development, apply the suitable trading and exchequers and patterns, assure effectiveness of training educations, innovatively and comprehensively. And finally improve the physical education level. Besides that the building of professional sport areas and equipment to satisfy the demand of physical practice, make student enjoy their physical practice with relaxing motion and profit from boring content. 


\section{References}

[1] Hong Yixing. "Sunshine Sports” dancing in the big recess--“Sunshine sport” practice and reelection on sport activities, Reading, writing, arithmetic-quality educational forum, 2015(3):89-90.

[2] He Faying. Participating in the construction of sports interactive platform is an effective way to promote the development of college sports in Minority Areas\& Liangshan Yi Autonomous Prefecture as an example. Journal of Southwestern Normal University (Natural Science Edition), 2014(11):147-151.

[3] Tao Lili. The explosion of the new mode of higher vocational college sports news professional literature appreciation course teaching -- Taking Guangxi College of Physical Education as an example, Northern Literature (published in late), 2014(4):198.

[4] Ma Yong, RaoPing, Zhou Jiansheng, Chen Hang. Exploration on teaching reform of sports humanistic sociology in Local Normal Colleges -- Based on the thinking of "the outline of the teaching of Humanities and sociology of sports", Contemporary sports science and technology, 2014, 4(23):106-107.

[5] Chen Wei,Shi Xiaoqiang, Chen $\mathrm{Xi}$, Li Bin. Construction of evaluation index system of employment quality of Sports Management Major Graduates--Taking the 12 sports colleges in our country as an example, Journal of Shanghai Institute of Physical Education, 2015, 39(3):30-33.

[6] Yang Zhigang, Zhang Li , Wang Li Na. Empirical study on the orientation and development strategy of the city sports culture in the international sports city of Shanghai, Journal of Nanjing Sport Institute (Social Science Edition), 2015(2):29-36.

[7] SunXiaolong, Zha Jianfang, Zahng Deli. On the issue of "learning and training" of the high level sports teams in the context of the construction of sports power -- Based on the investigation of 7 universities in Anhui Province, Journal of Anhwei Normal University (Natural Science Edition), 2015,38(6):598-602. 